\title{
Integral Resonant Control of a Piezoelectric Tube Actuator for Fast Nanoscale Positioning
}

\author{
B. Bhikkaji and S. O. Reza Moheimani, Senior Member, IEEE
}

\begin{abstract}
There is a need, in the wide ranging scientific community, to perform fast scans using scanning tunneling microscopes (STMs) and atomic force microscopes (AFMs) with nanoscale precision. In this paper, a piezoelectric tube of the type typically used in STMs and AFMs is considered. The resonant mode that hampers the fast scanning is identified and damped using a feedback control technique known as integral resonant control (IRC). The piezoelectric tube is then actuated to perform fast and accurate scans. IRC is a new feedback control technique suitable for damping highly resonant structures. Here, the IRC control technique is suitably modified to damp the resonance of a piezoelectric tube and achieve fast tracking of a wideband set point.
\end{abstract}

Index Terms-Control systems, piezoelectric transducers, vibration control.

\section{INTRODUCTION}

$\mathbf{S}$ CANNING tunneling microscopes (STMs) and atomic force microscopes (AFMs), when used at extreme magnifications, are capable of generating topographical maps of solid surfaces at micro to atomic resolution. In many STMs and AFMs, a probe is placed in close proximity, typically a few nanometers, to the material surface for which a topographical map is desired. The given material surface is scanned by moving the sample in a raster pattern, so that the probe interacts with the entire region of interest [10]. In general, scanning is performed by placing the sample on top of a piezoelectric tube and actuating the tube in a raster pattern.

Dynamics of piezoelectric tubes can be well approximated, under certain experimental conditions, by linear models [1], [19], [33], [35], [37]-[39]. The linear models normally reveal the presence of lightly damped resonance modes that make the piezoelectric tubes susceptible to mechanical vibrations. Nonlinear phenomenon such as creep and hysteresis can also be observed, when actuating the tube using low-frequency and highamplitude inputs, respectively, [24], [25]. In such scenarios, the linear approximations become inadequate. The objective of this paper is to perform fast scans using a piezoelectric tube. Current scanning frequencies are limited to less than $0.01 f_{r}$, where $f_{r}$ is the first resonance frequency of the piezoelectric tube. In this paper, the piezoelectric tube is scanned at frequencies comparable

Manuscript received December 14, 2007; revised April 23, 2008. Current version published October 8, 2008. Recommended by Technical Editor I-M. Chen. This work was supported in part by the Australian Research Council and the Centre for Complex Dynamic Systems and Control and in part by the Laboratory for Dynamics and Control of Nanosystems, University of Newcastle.

The authors are with the School of Electrical Engineering and Computer Science, University of Newcastle, Callaghan, NSW 2308, Australia (e-mail: reza.moheimani@newcastle.edu.au).

Color versions of one or more of the figures in this paper are available online at http://ieeexplore.org.

Digital Object Identifier 10.1109/TMECH.2008.2001186 to $0.1 f_{r}$. The main impediments to such scans are the presence of mechanical vibrations and hysteresis.

Slow scans are fast becoming a bane to the users of AFMs. Slow scans, though accurate, lead to inordinate waiting periods before an image can be obtained. Moreover, fast scans are also necessary to study fast processes in many diverse areas of science [16], [27], [28]. A good case for the design of control systems to perform fast scans is presented in [2].

Mechanical vibrations can be compensated by designing suitable feedback controllers to damp the resonant modes. This has been done by several authors in different contexts [1], [3], [7], [8], [12], [22], [30], [32], [33], [37]. The feedback controllers designed in all the aforementioned papers, though have been very effective, are very hard to design. Their design methodology may require the knowledge of advanced mathematics, and in some cases, they may warrant the solution of a nonconvex optimization problem, thereby making them computationally complex. Other active damping controllers such as positive position feedback (PPF) [23] and direct velocity feedback (DVF) [13] have also been used to damp flexible structures. DVF needs the implementation of a differentiator, and has the tendency to amplify high-frequency noises that come into picture when using external sensors. In the context of piezoelectric tubes, PPF can be designed using standard root-locus technqiue. However, as it is of second order, determination of three parameters from the root locus is not tractable [29]. A new feedback scheme, called integral resonant control (IRC), was introduced in [9]. In this scheme, an integral controller was used to damp the resonant modes of a cantilever beam. IRC is relatively straightforward and easy to design and implement. The computational complexity involved in their design is also insignificant. In this paper, this scheme is modified to damp the resonant mode of a piezoelectric tube, resulting in fast tracking of a high-bandwidth set point with nanoscale precision.

Nonlinearity in the form of hysteresis becomes visible when piezoelectric tubes are actuated using voltage signals of high amplitudes. At low-range scans (i.e., when actuating a piezoelectric tube with voltage signals of low amplitude), hysteresis can be neglected. A popular approach to compensate for hysteresis is to model it as a nonlinear function $H(\cdot)$, and then, eliminate it by cascading its inverse $H^{-1}(\cdot)$ with piezoelectric tube actuator [17], [20], [36], [40]. Feedback control schemes have also been used to compensate for hysteresis in [15], [33], and [37]. Since the late 1980s, it has been known that actuating piezoelectric transducers with current or charge sources rather than voltage sources significantly reduces hysteresis [34]. In fact, it has been noted that using current or charge sources, at least a fivefold reduction in hysteresis can be achieved [26]. Creep is another nonlinearity that occurs when low-frequency 
signals are used for actuating piezoelectric materials [14], [21]. Creep is not dealt within this paper, as this paper is about fast actuation of a piezoelectric tube.

Nonlinear modeling of hysteresis has always been a tedious task. Even in cases where the hysteresis is rate-independent, constructing a good model is both complex and computationally involved [31]. In cases where the hysteresis is rate-dependent, as in the case of piezoelectric materials, current modeling schemes offer little help. Use of charge or current sources does not necessitate the modeling of hysteresis. However, herein, the problem lies with the instrumentation of the charge or current sources capable of driving highly capacitive loads, as they are not commercially available at present. Invariably, the only resort is to make a "home made" charge or current source. The authors in [4] and [5] designed a dc-accurate charge source for the general purpose of exciting piezoelectric actuators without encountering hysteresis. In this paper, this charge amplifier is used for applying signals to the piezoelectric tube in order to avoid hysteresis.

This paper is formatted as follows. In the next section, the control scheme presented in [9] is discussed from the perspective of using it for a piezoelectric tube. In Section III, a piezoelectric tube of the type typically used in STMs and AFMs is considered, and its resonance mode is identified and damped using the scheme presented in Section II. The tube is also actuated in a raster pattern in Section III.

\section{Integral Resonant Control of A Piezoelectric TUBE ACTUATOR}

Piezoelectric tubes, like most other flexible structures, have an infinite number of resonant modes. However, from a computational perspective, and for practical control design purposes, one has to consider only a finite number of modes. Most piezoelectric tubes used in STMs and AFMs would have their first resonant mode within a bandwidth of $1 \mathrm{kHz},{ }^{1}$ while the other resonances would lie beyond $2 \mathrm{kHz}$ [1], [12], [19], [38], [39]. In order to actuate the piezoelectric tube at a rate of $0.1 f_{r}$, where $f_{r}$ is the first resonance frequency, it is enough to consider the first resonance alone. Therefore, here, the resonances beyond the first are neglected while modeling.

The transfer functions characterizing the dynamics of piezoelectric tubes, up to a bandwidth of $2 f_{r} \mathrm{~Hz}$, are typically of the form

$$
G(s)=\frac{\Gamma}{s^{2}+2 \zeta_{p} \omega_{p} s+\omega_{p}^{2}}
$$

where $\Gamma>0, \omega_{p}$ denotes the natural frequency, and $\zeta_{p}$ denotes the damping coefficient [1], [12], [19], [38]. Since the piezoelectric tubes are flexible structures, with poles close to the

\footnotetext{
${ }^{1}$ The actual resonance frequencies depend on the physical dimensions of the tube. It is possible to use small tubes that have very high resonance frequencies. However, most commercially available STMs and AFMs use relatively large tubes. This is needed to scan large areas, e.g., $100 \mu \mathrm{m} \times 100 \mu \mathrm{m}$. Such tubes have relatively large frequencies.
}

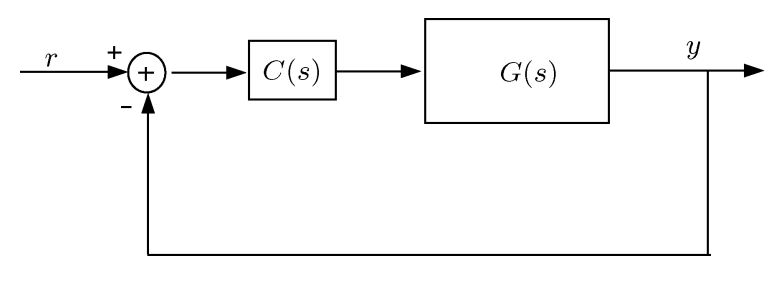

Fig. 1. Closed-loop system with the integral controller $C(s)=(K / s)$ around $G(s)$.

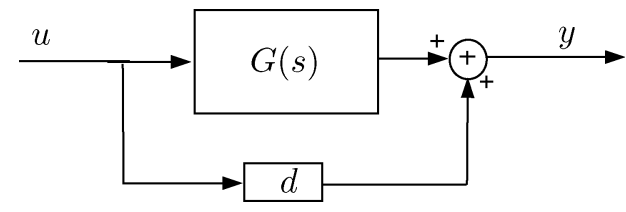

Fig. 2. Transfer function $\bar{G}(s)$ obtained by adding a $d$ to $G(s)$.

imaginary axis, (1) can be approximated by

$$
G(s)=\frac{\Gamma}{s^{2}+\omega_{p}^{2}} .
$$

Wrapping an integral controller $C(s)=(K / s)$ around $G(s)$, [see (2) and Fig. 1], would lead to a closed-loop system of the form

$$
G^{(c l)}(s)=\frac{G(s) C(s)}{1+G(s) C(s)} .
$$

It can be checked that the poles of (3) are the roots of the polynomial

$$
p(s)=s\left(s^{2}+\omega_{p}^{2}\right)+K \Gamma .
$$

This implies that

$$
p(\mathrm{i} \omega)=\mathrm{i} \omega\left(-\omega^{2}+\omega_{p}^{2}\right)+K \Gamma
$$

can never satisfy the Hermite-Biehler theorem (HB theorem), $[11$, p. 41]. Hence, $p(s)$ can never be Hurwitz for any $K>$ 0 . Alternatively stated, $p(s)$ can never be stabilized using the integral controller.

Consider the transfer function

$$
\bar{G}(s)=\frac{\Gamma}{s^{2}+\omega_{p}^{2}}+d
$$

which is obtained by adding a feedthrough $d$ term to (2) (see Fig. 2). Note that wrapping an integral controller $C(s)=(K / s)$ around $\bar{G}(s)$ would lead to the closed-loop system (see Fig. 3)

$$
\begin{aligned}
\bar{G}^{(c l)}(s) & =\frac{\bar{G}(s)(K / s)}{1+(K / s) \bar{G}(s)} \\
& =\frac{K d\left(s^{2}+\omega_{p}^{2}+(\Gamma / d)\right)}{s\left(s^{2}+\omega_{p}^{2}\right)+K d\left(s^{2}+\omega_{p}^{2}+(\Gamma / d)\right)} .
\end{aligned}
$$

It can also be checked that the closed-loop system corresponding to the plant input and output [i.e., with respect to $G(s)$ ] is given by

$$
G^{(c l)}(s)=\frac{G(s)(K / s)}{1+(K / s) \bar{G}(s)}
$$




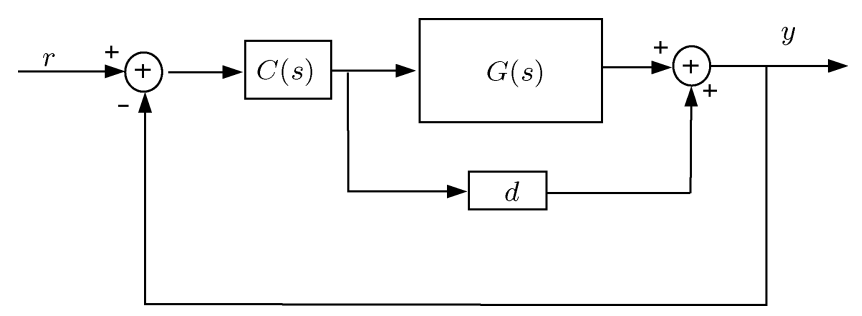

Fig. 3. Closed-loop system with the integral controller $C(s)=(K / s)$ around $\bar{G}(s)$.

The poles of (7) and (8) are the roots of the polynomial

$$
\bar{p}(s)=s\left(s^{2}+\omega_{p}^{2}\right)+K d\left(s^{2}+\omega_{p}^{2}+\frac{\Gamma}{d}\right)
$$

which implies

$$
\bar{p}(\mathrm{i} \omega)=\mathrm{i} \omega\left(-\omega^{2}+\omega_{p}^{2}\right)+K d\left(-\omega^{2}+\omega_{p}^{2}+\frac{\Gamma}{d}\right) .
$$

For (9) to satisfy the Hermite-Biehler theorem, the absolute value of the roots of the imaginary part of (10) must be less than the absolute value of the roots of the real part. Furthermore, the product $K d$ has to be positive. In other words,

$$
0<\omega_{Z}<\omega_{P}
$$

where

$$
\omega_{Z}=\sqrt{\omega_{p}^{2}+\frac{\Gamma}{d}}
$$

and

$$
\omega_{P}=\omega_{p}
$$

and $K d>0$. It can be inferred that for (11) to hold, $d$ has to be negative. And hence, $K<0$ for $K d>0$ to hold. A preferred position for $\omega_{Z}$ would be to lie in the midpoint between the origin and $\omega_{P}$, which is achieved when

$$
d=-\frac{4 \Gamma}{3 \omega_{p}^{2}} .
$$

Note that the static gain of (8) is given by

$$
\left|G^{(c l)}(0)\right|=\left|\frac{\Gamma}{d\left(\omega_{p}^{2}+\frac{\Gamma}{d}\right)}\right| .
$$

This implies that the static gain would be equal to 1 , or $0 \mathrm{~dB}$ if $d=\left(-2 \Gamma / \omega_{p}^{2}\right)$.

It does not escape our notice that (8) boils down to

$$
\begin{aligned}
G^{(c l)}(s) & =\frac{G(s)(K / s)}{1+(K / s) \bar{G}(s)} \\
& =\frac{G(s)(K / s)}{1+(K / s)(G(s)+d)} \\
& =\frac{G(s)(K / s)}{1+(K / s) G(s)+d(K / s)} \\
& \triangleq \frac{G(s) \bar{C}(s)}{1+G(s) \bar{C}(s)}
\end{aligned}
$$

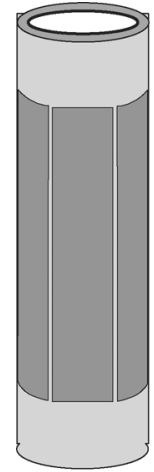

(a)

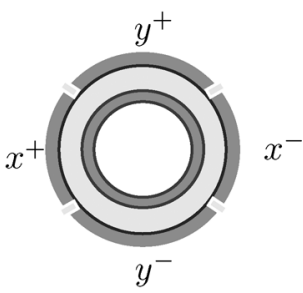

(b)
Fig. 4. Illustration of the piezoelectric tube. (a) Side view. (b) Top view.

where

$$
\begin{aligned}
\bar{C}(s) & \triangleq \frac{K}{s}\left(1+d \frac{K}{s}\right)^{-1} \\
& =\frac{K}{s+K d} .
\end{aligned}
$$

In other words, the whole scheme can be implemented in a standard feedback setup, as shown in Fig. 1 with $C(s)$ replaced by $\bar{C}(s)$. However, using a lossy integrator $\bar{C}(s)$ directly and achieving the damping does not provide any insight into the working of the control scheme. In particular, it tells very little about the swapping of the zeros that is achieved by adding the $d$ term.

\section{ACtuation of A Piezoelectric Tube}

A piezoelectric tube is a thin-walled cylindrical tube made of piezoelectric material. The inner and outer walls of the tube are coated with a layer of silver. The silver coating acts as the electrodes of the piezoelectric tube. The outer electrode of the piezoelectric tube scanner is axially quartered into four equal sections. A pair of opposite quartered electrodes are referred to as the $x-x$ electrodes and the other pair is referred to as the $y-y$ electrodes (see Fig. 4). Good illustrations of the tube can be found in [5], [10], [18], and [12].

\section{A. Experimental Setup}

In the experimental setup used here, a piezoelectric tube is held upright with its bottom glued to a rigid surface. A small aluminum cube is bonded to the upper end of the tube. This cube represents the seat where the materials that need to be scanned are placed. The head of an ADE Technologies 4810 capacitive sensor is placed in close proximity to the face of aluminum cube along the $x$-axis (see Fig. 5). The inner electrode of the piezoelectric tube is grounded. An electrode from the $x-x$ pair, referred as the $x^{+}$electrode, is chosen as the input end of the piezoelectric tube. The whole setup consisting of the piezoelectric tube with the bonded aluminum cube and the heads of the capacitive sensors is placed in a specially constructed 


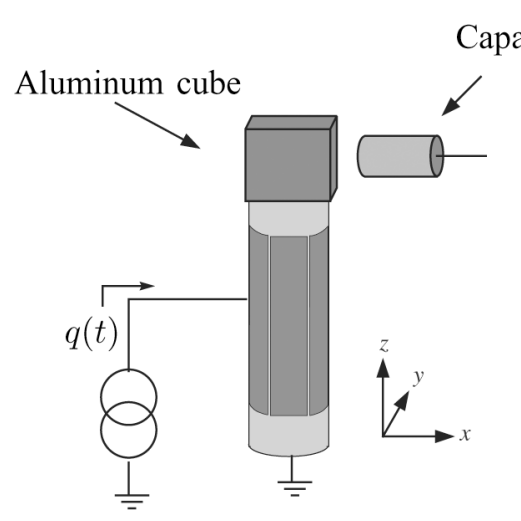

Fig. 5. Schematic diagram of the experimental setup.

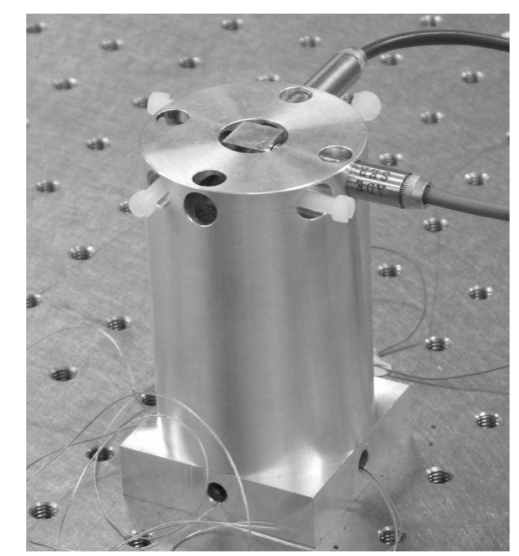

Fig. 6. Piezoelectric tube mounted inside an aluminum shield. The $x$-axis capacitive sensor is shown secured at right angles to a cube mounted onto the tube tip, and the $y$-axis capacitive sensor is secured at right angles to the perpendicular face of the aluminum cube.

cylindrical enclosure (refer to Fig. 6). The cylindrical enclosure protects the experimental setup from external noise.

As mentioned in Section I, the goal is to actuate this piezoelectric tube in a raster pattern. A desired trajectory for the piezoelectric tube would be to repeatedly trace straight lines back and forth in the $x$-direction, while slowly increasing its position in the $y$-direction. A common practice to track such a trajectory is to apply a triangular waveform to the $x^{+}$electrode and a "very slowly" increasing ramp signal to the $y^{+}$(one of the electrodes of the $y-y$ pair, see Fig. 4). In fact, to have a good scan of the surface the changes in $y$-direction must be "quasi-static" with respect to the changes in the $x$-direction. Normally, for illustration purposes, the slowly varying ramp in the $y^{+}$electrode is either replaced by a dc signal or assumed to be earthed or open circuited (see [1], [19], and [33]). Here, it is earthed.

When a signal is applied at the electrode $x^{+}$, the piezoelectric tube deforms causing a change in the capacitance between the aluminum cube and the head of the capacitive sensor. The change in the capacitance is used by the capacitive sensor to measure the distance between its head and the aluminum cube. This distance, denoted by $D(t)$, is also recorded as an output. In summary, the piezoelectric tube is modeled as a SISO system

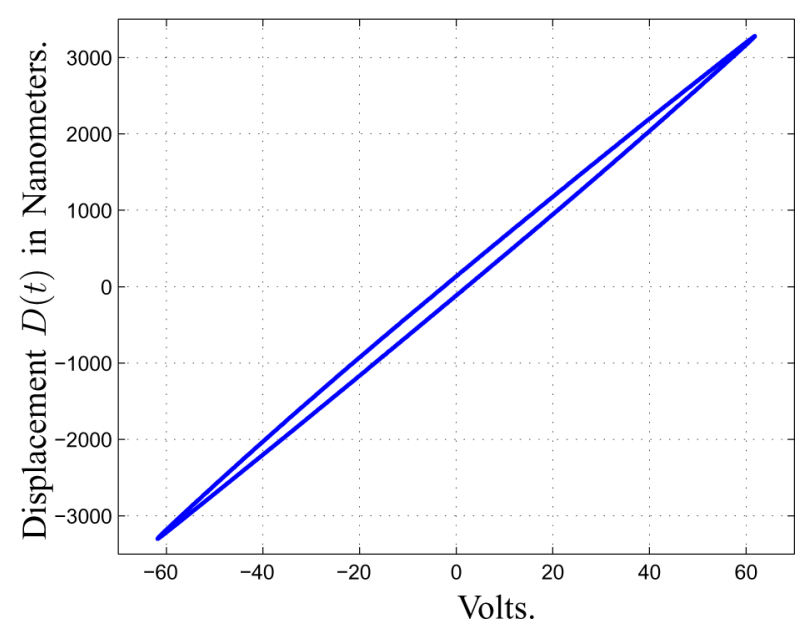

Fig. 7. Response $D(t)$ recorded by the capacitive sensor to a sinusoidal input of $5-\mathrm{Hz}$ frequency and 62-V amplitude at the $x^{+}$electrode.

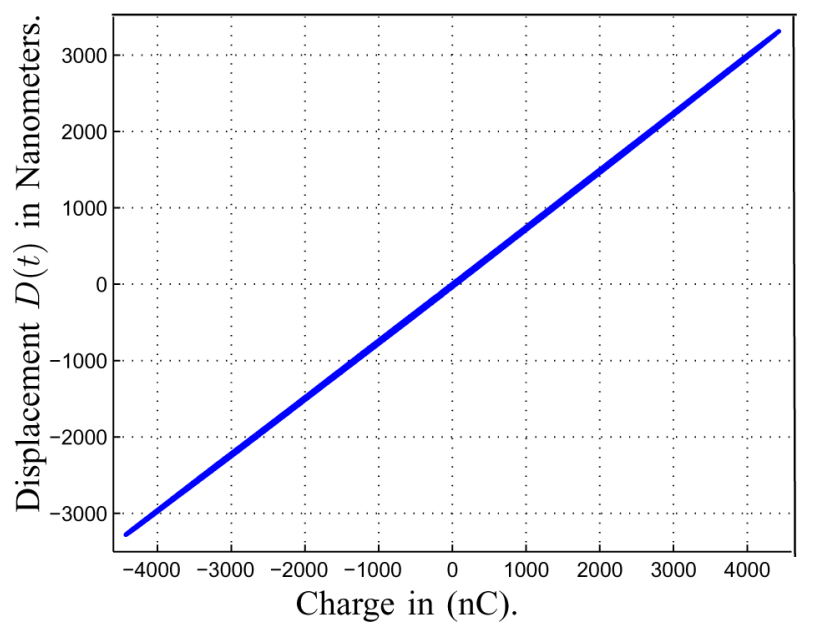

Fig. 8. Response $D(t)$ recorded by the capacitive sensor to a sinusoidal input of 5- $\mathrm{Hz}$ frequency and 4500-nC amplitude at the $x^{+}$electrode.

with the input being the signal applied at the $x^{+}$electrode and the output being the capacitive sensor measurement $D(t)$.

In this paper, charge signals $q(t)$, or signals obtained by modulating charges instead of voltages, are used as inputs to avoid hysteresis. In Figs. 7 and 8, responses recorded by the capacitive sensor to sinusoidal waveform inputs $v_{1}(t)$ and $q_{1}(t)$, respectively, with a frequency of $5 \mathrm{~Hz}$ are plotted. It can be noted that $v_{1}(t)$ has amplitude of about $62 \mathrm{~V}$ and $q_{1}(t)$ has an amplitude of $4500 \mathrm{nC}$. More importantly, it can be noted that the response to the charge signal is linear while the response recorded for the voltage signal $v_{1}(t)$ is nonlinear.

\section{B. Identification and Control}

Using the charge amplifier, swept sine waves are applied as input at the $x^{+}$electrode of the piezoelectric tube. Application of the swept sine input causes the tube to bend. The input $q(t)$, applied to the $x^{+}$electrode, and the corresponding capacitive sensor output $D(t)$ are fed into a spectrum analyzer, which computes the frequency response function (FRF) 

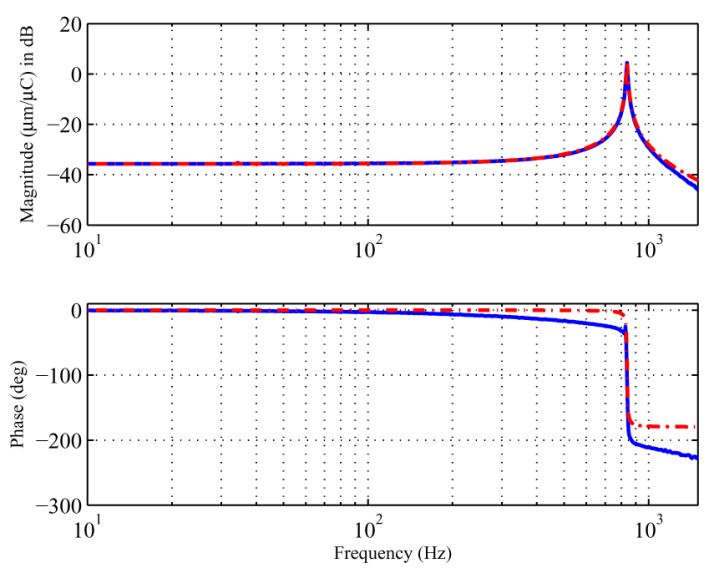

Fig. 9. Magnitude plots of the data (solid) along with the corresponding parametric model (dashed dots).

TABLE I

EMPIRICAL TRANSFER FUNCTION PARAMETERS

\begin{tabular}{|l|l|}
\hline Name & Value \\
\hline$\Gamma$ & $4.6079 \times 10^{5}$ \\
$2 \sigma_{p} \omega_{p}$ & 75.3 \\
$\omega^{2}$ & $2.733 \times 10^{7}$ \\
\hline
\end{tabular}

$G_{D q}(\mathrm{i} \omega)=(D(\mathrm{i} \omega) / q(\mathrm{i} \omega))$, where $D(\mathrm{i} \omega)$ and $q(\mathrm{i} \omega)$ are the Fourier transform of $D(t)$ and $q(t)$, respectively, a set of desired frequency points. The computed FRF is plotted in Fig. 9. The model

$$
G_{D q}(s)=\frac{\Gamma}{s^{2}+2 \sigma_{p} \omega_{p} s+\omega_{p}^{2}}
$$

with the parameters tabulated in Table I was fit for the FRF data (see Fig. 9). It is apparent that the model captures the magnitude response, with good accuracy, but not the phase response. The phase response of the system suggests a delay in the system response. This delay is due to the presence of a zero between the first and the second resonance (the second resonance is not seen in Fig. 9, as it is out of the bandwidth of interest), which cannot be captured using a second-order model. In Fig. 12(a), the responses $D(t)$ recorded by the capacitive sensor to the triangular waveform inputs with fundamental frequencies 10, 40, and $80 \mathrm{~Hz}$ are plotted. It is apparent that the recorded responses are not triangular waveforms. This distortion is due to the amplification of the harmonics of these triangular waveforms that lie close to the resonant mode. In the case of the $10 \mathrm{~Hz}$ triangular waveform it the ninth harmonic, while it is the fifth and the third harmonic, respectively, in the cases of 40 and $80 \mathrm{~Hz}$ triangular waveforms.

As mentioned earlier, to design the control scheme, $G_{D q}(s)$ is approximated by setting $2 \sigma \omega$ in (18) to zero, and $d=-\left(2 \Gamma / \omega_{p}^{2}\right)$ is added to obtain

$$
\bar{G}_{D q}(s)=\frac{\Gamma}{s^{2}+\omega_{p}^{2}}+d .
$$

Note that for the chosen value of $d$, the closed-loop system (8) will have a unit static gain. In Fig. 10, the root locus of $\left(\bar{G}_{D q}(s) / s\right)$, or alternatively, the locus of the poles of $r(s)=s\left(s^{2}+\omega_{p}^{2}\right)+K d\left(s^{2}+\omega_{p}^{2}+(\Gamma / d)\right)$, for $K<0$,

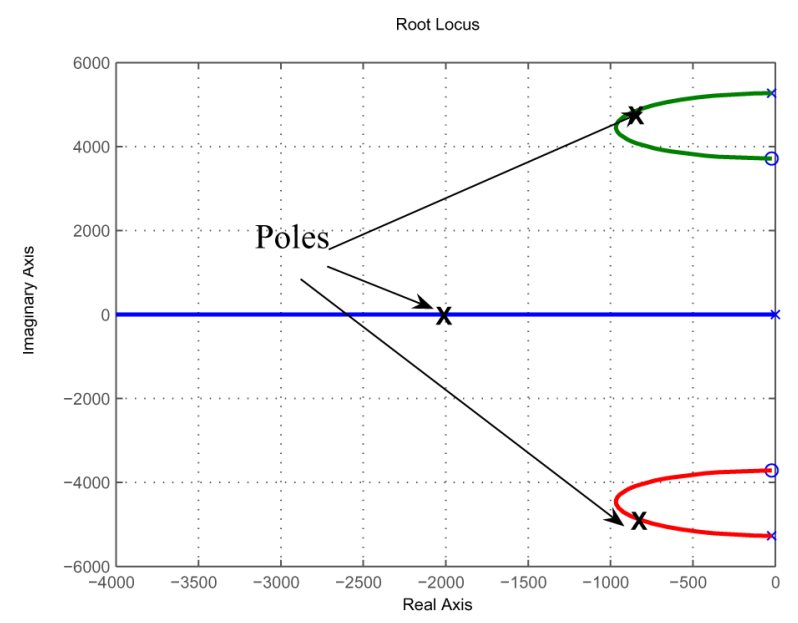

Fig. 10. Root locus of the plot of $\left(\bar{G}_{D q}(s) / s\right)$ for $K<0$. $\mathbf{X}$ denotes the closed-loop poles of $G^{(c l)}(s)$ for a gain of $K=-10^{5}$.
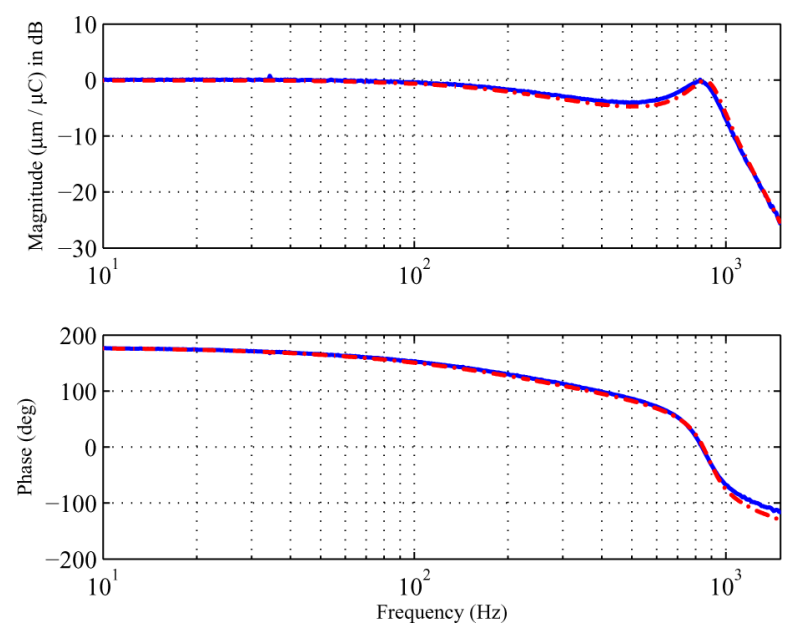

Fig. 11. Predicted (solid) and the experimentally determined (dashed) magnitude responses of the closed-loop system.

is plotted. Here, the gain of the integral controller is set to $K=-10^{5}$. In Fig. 11, the magnitude response of $G^{(c l)}(\mathrm{i} \omega)$, (8), is plotted along with experimentally determined magnitude response. It can be observed from the plot that the experimentally determined magnitude response matches predicted magnitude response. Here, a dSPACE 1103 PPC controller board is used for the real-time implementation of the feedthrough term $d$ and the integral controller. A sampling frequency of $20 \mathrm{kHz}$ was used to avoid aliasing. Simulink was used to download the feedthrough term and the controller in to the dSPACE board. The gain $K=-10^{5}$ is not optimal in any particular sense. As the goal is to have a good damping, an optimal choice would be a $K$ that gives maximum modal damping. It can be shown that this is achieved by selecting $K_{\mathrm{opt}}=-\left(\omega_{P} / d\right) \sqrt{\left(\omega_{P} / \omega_{Z}\right)}$ [6], where $\omega_{Z}, \omega_{P}$, and $d$ are as defined in (12), (13), and (14), respectively. Note that for $d=-\left(4 \Gamma / 3 \omega_{p}^{2}\right),(14), \omega_{Z}=\left(\omega_{P} / 2\right)$. Which implies $K_{\mathrm{opt}}=\left(\omega_{P} / d\right) \sqrt{2} \approx 3.3 \times 10^{5}$. It was found that this gain was too large to implement, and the charge amplifier used in the experiments could not generate the necessary 

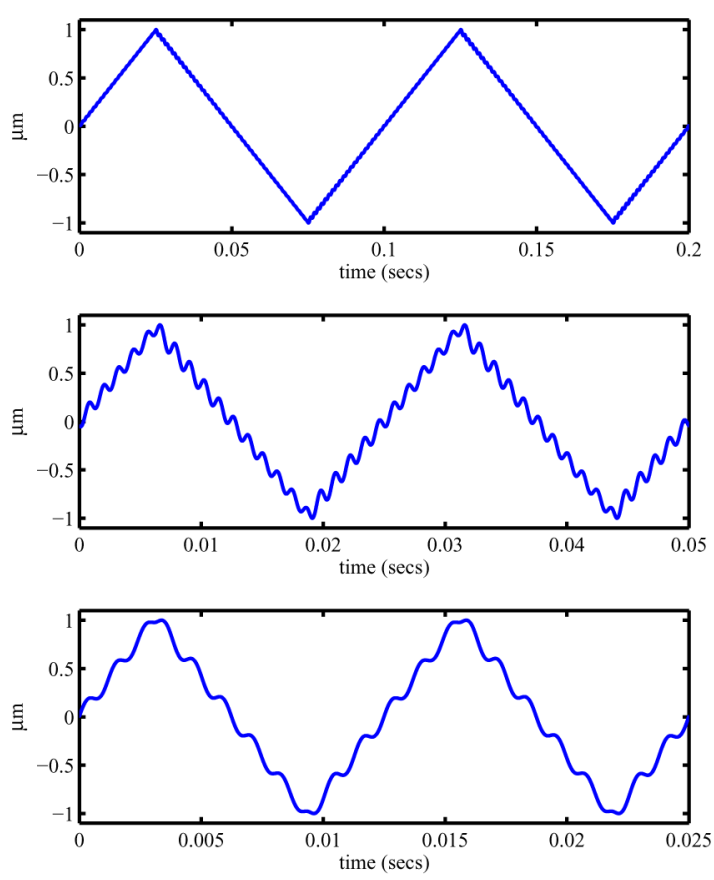

(a)
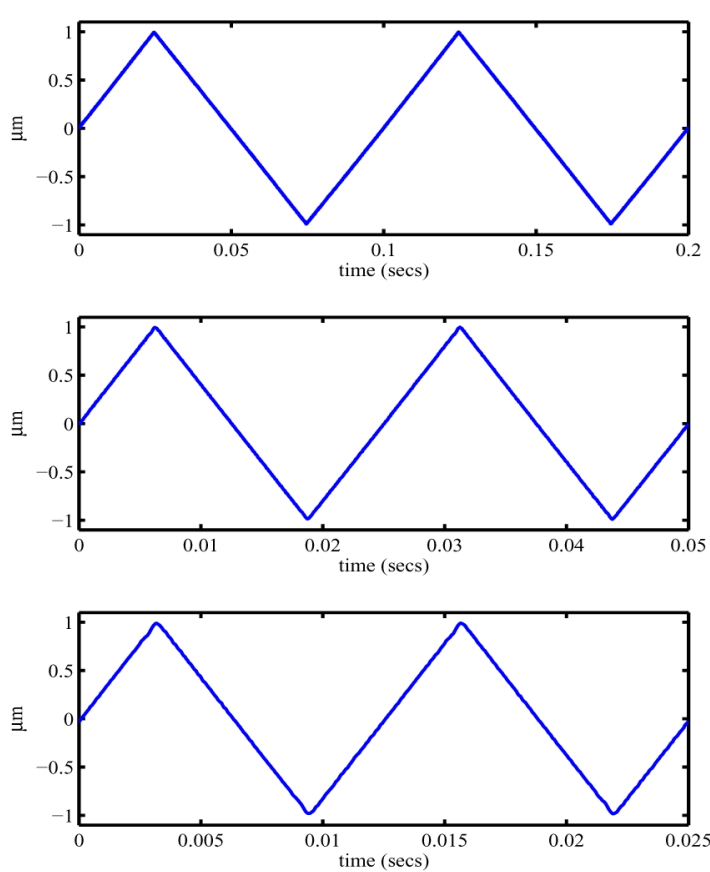

(b)

Fig. 12. (a) Response $D(t)$, recorded by the capacitive sensor to triangular waveform inputs with fundamental frequencies 10,40 , and $80 \mathrm{~Hz}$, respectively. (b) Response recorded by the capacitive sensor, in closed-loop, for inputs of the form $u(t)$, (20), for $f_{d}(t)$ 's with fundamental frequencies 10 , 40, and $80 \mathrm{~Hz}$, respectively.

control signal and went into saturation. Hence, a suboptimal $K=-10^{5}$ was chosen.

In order to actuate the piezoelectric tube in a raster pattern, an input of the form

$$
u(t) \triangleq \sum_{k=1}^{\infty} \frac{a_{k}}{\left|G_{d q}^{(c l)}\left(\mathrm{i} \omega_{k}\right)\right|} \sin \left(\omega_{k} t-\phi_{k}\right)
$$

where $a_{k}$ and $\omega_{k}$ are such that

$$
f_{d}(t) \triangleq \sum_{k=1}^{\infty} a_{k} \sin \left(\omega_{k} t\right)
$$

is the desired triangular waveform output $D(t)$ at the capacitive sensor in the Fourier series form and

$$
\phi_{k} \triangleq \arg G_{d q}^{(c l)}\left(\mathrm{i} \omega_{k}\right)
$$

is applied at the $x^{+}$electrode. It is easy to see that applying $u(t)$ to closed-loop plant should give a triangular waveform, provided $G^{(c l)}(\mathrm{i} \omega)$ models the frequency response of the closedloop plant with reasonable accuracy. It is apparent from Fig. 11 that closed-loop model $G_{d q}^{(c l)}(s)$, (8), fits the frequency response of the closed-loop plant with reasonable accuracy. In Fig. 12, the capacitive sensor response $D(t)$ recorded for inputs of the form $u(t),(20)$, with $f_{d}(t)$ being triangular waveforms of 10,40 , and $80 \mathrm{~Hz}$, respectively. It can be observed that the capacitive sensor responses appear triangular. In the following, the rms errors in the capacitive sensor outputs (plotted in Fig. 12) are estimated using the approximation

$$
\begin{aligned}
\epsilon_{\mathrm{rms}} & \triangleq \sqrt{\frac{1}{T} \int_{0}^{T}\left[f_{d}(t)-f_{o}(t)\right]^{2} d t} \\
& \approx \sqrt{\frac{1}{L h} \sum_{k=0}^{L-1}\left[f_{d}(k h)-f_{o}(k h)\right]^{2} h} \\
& =\sqrt{\frac{1}{L} \sum_{k=0}^{L-1}\left[f_{d}(k h)-f_{o}(k h)\right]^{2}} .
\end{aligned}
$$

In (23), $f_{d}(t)$ denotes the desired triangular waveform, $f_{o}(t)$ denotes the capacitive sensor output, $T$ denotes the period of the desired triangular waveform, and $h$ denotes the sampling rate. Note that the desired triangular waveforms $f_{d}(t)$ 's corresponding capacitive sensor outputs $f_{o}(t)$ 's (plotted in Fig. 12) have an amplitude of $1 \mu \mathrm{m}$ with fundamental frequencies being 10,40 , and $80 \mathrm{~Hz}$, respectively. The estimates of the $\epsilon_{\mathrm{rms}}$, with $h=5 \times 10^{-5} \mathrm{~s}$, for the 10,40 , and $80 \mathrm{~Hz}$ triangular waveforms were found to be $4.9,9$, and $15.6 \mathrm{~nm}$, respectively. Not surprisingly, the rms error increases with the increasing frequency of scan. Since high-frequency triangular waveforms excite the unmodeld higher order harmonics, it is general practice to consider only $80 \%$ of the scan area. In other words, $20 \%$ of the data around the corners, i.e., $20 \%$ data close to the peaks in Fig. 12, are discarded, since these regions correspond to the higher hormonics that excite the unmodeled dyanamics. The $\epsilon_{\mathrm{rms}}$ estimates of the $80 \%$ scans, for 10,40 , and $80 \mathrm{~Hz}$ triangular waveforms, were found to be $4.7,7.5$, and $12.5 \mathrm{~nm}$, respectively. 


\section{CONCLUSION}

In this paper, a piezoelectric tube of the type used in STMs and AFMs was considered. The objective was to actuate this tube at high scanning frequencies with nanoscale precision. It was noted that the main impediment to perform fast actuation is the presence of a resonant mode. This resonant mode was damped by designing a suitable IRC controller in the feedback. Damping of the resonance mode enabled vibration-free actuations with scanning frequencies comparable to $0.1 f_{r}$, where $f_{r}$ is the resonance frequency of the tube. The rms errors obtained for actuation with scanning frequencies 10, 40, and $80 \mathrm{~Hz}$ confirm that the scans are of nanoscale precision.

\section{ACKNOWLEDGMENT}

The authors wish to thank Dr. A. J. Fleming for designing and building the experimental setup, including the charge drive electronics, used in this paper.

\section{REFERENCES}

[1] A. Daniele, S. Salapaka, M. V. Salapaka, and M. Daleh, "Piezoelectric tubes for atomic force microscopes: Design of lateral sensors, identification and control," in Proc. Amer. Control Conf., San Diego, CA, 1999, pp. 253-257.

[2] P. K. Hansma, G. Schitter, G. E. Fantner, and C. Prater, "High-speed atomic force microscopy," Science, vol. 314, no. 3799, pp. 601-602, 2006.

[3] A. J. den Hamer, G. Z. Angelis, and N. B. Roozen, "Broad-band active vibration supression using PPF focused on industrial application," IEEE/ASME Trans. Mechatronics, vol. 10, no. 2, pp. 146-152, Apr. 2005.

[4] A. J. Fleming and S. O. R. Moheimani, "Precision current and charge amplifiers for driving high capacitive piezoelectric loads," Electron. Lett., vol. 39, no. 3, pp. 282-284, Feb. 2003.

[5] A. J. Fleming and S. O. R. Moheimani, "Sensorless vibration supression and scan compensation for piezoelectric tube nanopositioners," IEEE Trans. Control Syst. Technol., vol. 14, no. 1, pp. 33-44, Jan. 2006.

[6] A. Preumont, B. de Marneffe, A. Deraemaeker, and F. Bossens, "The damping of a truss structure with a piezoelectric transducer," Comput. Struct., vol. 86, pp. 227-239, 2008.

[7] S. S. Aphale, B. Bhikkaji, and S. O. R. Moheimani, "Minimizing scanning errors in piezoelectric stack-actuated nanopositioning platforms," IEEE Trans. Nanotechnol., vol. 7, no. 1, pp. 79-90, Jan. 2008.

[8] S. S. Aphale, A. J. Fleming, and S. O. R. Moheimani, "High speed nanoscale positioning using a piezoelectric tube actuator with active shunt control," Micro Nano Lett., vol. 2, no. 1, pp. 9-12, Jan. 2007.

[9] S. S. Aphale, A. J. Fleming, and S. O. R. Moheimani, "Integral resonant control of collocated smart structures," Smart Mater. Struct., vol. 16, pp. 439-446, 2007.

[10] B. Bhushan, Ed., Springer Handbook of Nanotechnology. Heidelberg, Germany: Springer-Verlag, 2004.

[11] S. P. Bhattacharyya, H. Chapellat, and L. H. Keel, Robust Control: The Parametric Approach. $\quad$ NJ: Prentice-Hall, 1995.

[12] B. Bhikkaji, M. Ratnam, A. J. Fleming, and S. O. R. Moheimani, "Highperformance control of piezoelectric tube scanners," IEEE Trans. Control Syst. Technol., vol. 5, no. 5, pp. 853-866, Sep. 2007.

[13] C. W. deSilva, Vibration Fundamentals and Practice. Boca Raton, FL: CRC Press, 1999.

[14] D. Croft, G. Shedd, and S. Devasia, "Creep, hysteresis, and vibration compensation for piezoactuators: Atomic force microscopy application," in Proc. Amer. Control Conf., Chicago, IL, Jun. 2000, pp. 2123-2128.

[15] J. M. Cruz-Hernandez and V. Hayward, "Phase control approach to hysteresis reduction," IEEE Trans. Control Syst. Technol., vol. 9, no. 1, pp. 17-26, Jan. 2001.

[16] D. A. Walters, "An AFM for small cantilevers," Proc. SPIE, Micro Machining Imag., vol. 3009, pp. 43-47, 1997.
[17] D. Hughes and J. T. Wen, "Perisach modeling and compensation for smart material hysteresis," Proc. SPIE Active Mater. Smart Struct., vol. 2427, pp. 50-64, 1994.

[18] S. Devasia, E. Eleftheriou, and S. O. R. Moheimani, "A survey of control issues in nanopositioning," IEEE Trans. Control Syst. Technol., vol. 15, no. 5, pp. 802-823, Sep. 2007.

[19] G. Schitter and A. Stemmer, "Identification and open-loop tracking control of a piezoelectric tube scanner for high-speed scanning probe microscopy," IEEE Trans. Control Syst. Technol., vol. 12, no. 3, pp. 449-454, May 2004.

[20] P. Ge and M. Jouaneh, "Tracking control of a piezoelectric actuator," IEEE Trans. Control Syst. Technol., vol. 4, no. 3, pp. 209-216, May 1996.

[21] H. Jung, J. Y. Shim, and D. Gweon, "New open-loop actuating method of piezoelectric actuators for removing hysteresis and creep," Rev. Sci. Instrum., vol. 71, no. 9, pp. 3436-3440, 2000.

[22] I. N. Kar, K. Seto, and F. Doi, "Multimode vibration control of a flexible structure using $H_{\infty}$-based robust control," IEEE/ASME Trans. Mechatronics, vol. 5, no. 1, pp. 23-31, Mar. 2000.

[23] J. L. Fanson and T. K. Caughey, "Positive position feedback control for large space structures," AIAA J., vol. 28, no. 4, pp. 717-724, 1990.

[24] K. K. Leang and S. Devasia, "Hysteresis, creep, and vibration compensation for piezoactuators: Feedback and feedforward control," in Proc. 2nd IFAC Conf. Mechatronic Syst., Berkeley, CA, 2002, pp. 283-289.

[25] K. K. Leang and S. Devasia, "Iterative feedforward compensation of hysteresis in piezo positioners," in Proc. IEEE 42nd Conf. Decis. Control, Maui, HI, Dec. 2003, pp. 2626-2631.

[26] H. Kaizuka and B. Siu, "Simple way to reduce hysteresis and creep when using piezoelectric actuators," Jpn. J. Appl. Phys. 2, Lett., vol. 27, no. 5, pp. 773-776, May 1988.

[27] M. B. Viani, T. E. Schaffer, A. Chand, M. Rief, H. E. Gaub, and P. K. Hansma, "Small cantilevers for force spectroscopy of single molecules," J. Appl. Phys., vol. 86, no. 4, pp. 2258-2262, 1999.

[28] M. B. Viani, L. I. Pietrasanta, J. B. Thompson, A. Chand, I. C. Gebeshuber, J. H. Kindt, M. Richter, H. G. Hansma, and P. K. Hansma, "Probing protein-protein interactions in real time," Nature Struct. Biol., vol. 7, no. 8, pp. 644-650, 2000.

[29] M. Ratnam, B. Bhikkaji, A. Fleming, and S. O. R. Moheimani, "PPF control of a piezoelectric tube scanner," in Proc. 44th IEEE Conf. Decis. Control Eur. Control Conf. (ECC 2005), Seville, Spain, pp. 1168-1173.

[30] I. A. Mahmood, S. O. R. Moheimani, and B. Bhikkaji, "Precise tip positioning of a flexible manipulator using resonant control," IEEE/ASME Trans. Mechatronics, vol. 13, no. 2, pp. 180-186, Apr. 2008.

[31] I. Mayergoyz, Mathematical Models of Hysteresis and Their Applications. New York: Elsevier, 2003.

[32] S. O. R. Moheimani and B. J. G. Vautier, "Resonant control of structural vibration using charge-driven piezoelectric actuators," IEEE Trans. Control Syst. Technol., vol. 13, no. 6, pp. 1021-1035, Nov. 2005.

[33] N. Tamer and M. Daleh, "Feedback control of piezoelectric tube scanners," in Proc. 33rd Conf. Decis. Control, Lake Buena Vista, FL, 1994, pp. 18261831.

[34] C. V. Newcomb and I. Flinn, "Improving the linearity of piezoelectric ceramic actuators," Electron. Lett., vol. 18, no. 11, pp. 442-443, May 1982.

[35] O. M. El Rifai and K. Youcef-Toumi, "Coupling of piezoelectric tube scanning in scanning probe microscopes," in Proc. Amer. Control Conf., Arlington, VA, 2001, pp. 3251-3255.

[36] R. Venkataraman and P. S. Krishnaprasad, "Approximate inversion of hysteresis: Theory and numerical results," in Proc. 39th IEEE Conf. Decis. Control, 2000, pp. 4448-4454.

[37] S. Salapaka and A. Sebastian, "Control of nanopositioning device," in Proc. 42nd IEEE Conf. Decis. Control, Maui, HI, 2003, pp. 2644-2648.

[38] T. Ohara and K. Youcef-Toumi, "Dynamics and control of piezo tube actuators for subnanometer precision applications," in Proc. Amer. Control Conf., Seattle, WA, 1995, pp. 3808-3812.

[39] M. E. Taylor, "Dynamics of piezoelectric tube scanners for scanning probe microscopy," Rev. Sci. Instrum., vol. 64, no. 1, pp. 154-158, 1993.

[40] X. Tan, R. Venkataraman, and P. S. Krishnaprasad, "Control of hysteresis: Theory and experimental results," in Modeling, Signal Processing, and Control in Smart Structures, V. S. Rao, Ed. Bellingham, WA: SPIE, 2001, vol. 4326, pp. 101-112. 
B. Bhikkaji received the Ph.D. degree in signal processing from Uppsala University, Uppsala, Sweden, in 2004.

He is currently a Research Academic in the School of Electrical Engineering and Computer Science, University of Newcastle, Newcastle, Australia. His current research interests include system identification, signal processing, and active noise and vibration control of flexible structures.
S. O. Reza Moheimani (S'93-M'97-SM'00) received the Ph.D. degree in 1996 from the University of New South Wales, Australian Defense Force Academy, Canberra, Australia, where he also held a research position.

In 1997, he joined the University of Newcastle, Callaghan, Australia, where he is currently an Associate Professor in the School of Electrical Engineering and Computer Science, an Assistant Dean for Research (Engineering), the Head of the Laboratory for Dynamics and Control of NanoSystems, and the Associate Director of the Australian Research Council (ARC) Centre for Complex Dynamic Systems and Control, an Australian Government Centre of Excellence. He is the author or coauthor of two books, several edited volumes, and more than 150 refereed articles in the areas of robust control and estimation, smart structures, active noise and vibration control, mechatronic systems, and nanotechnology.

Dr. Moheimani has chaired a number of international workshops and conferences, and is an Associate Editor of several international journals including the IEEE TRANSACTIONS ON CONTROL SySTEMS TECHNOLOGY. 